\title{
Density Results for Energy Spaces on some Fractafolds
}

\author{
Maria Rosaria Lancia, Valerio Regis Durante and Paola Vernole
}

\begin{abstract}
In this paper we prove density results for the domains of energy forms defined on a scale irregular fractal surface $S^{(\xi)}$, as well as on the corresponding threedimensional bounded cylindrical domain $Q^{(\xi)}$, whose lateral boundary is $S^{(\xi)}$.
\end{abstract}

Keywords. Scale irregular fractal surfaces, density results, trace theorems, energy forms

Mathematics Subject Classification (2010). Primary 28A80, 46E35, secondary $31 \mathrm{C} 25$

\section{Introduction}

It is well known that industrial processes and natural phenomena take place across irregular media, fractals turn out to be good tools to model these irregular geometries.

Fractal curves are very often used in modeling physical phenomena (see e.g. [21,22]) and there is a huge literature on this topic. Classical fractal curves such as the Sierpiński gasket, the Koch curve and the snowflake are nice self similar sets and energy forms on these sets can be obtained as limits of suitable approximating energies by exploiting the self-similarity of the underlying set (see e.g. [5]). On scale irregular (non self-similar) sets, known as fractal mixture sets, energy forms can be defined too (see $[2,18]$ ).

Recently there has been a recent increasing interest towards applications involving fractal surfaces. To our knowledge the first examples of energies on fractal surfaces can be found in $[8-12,19]$, where the fractal surface is obtained

M. R. Lancia: Dipartimento di Scienze di Base e Applicate per l'Ingegneria, Università di Roma Sapienza, Via A. Scarpa 16, 00161 Roma, Italy;

mariarosaria.lancia@sbai.uniroma1.it

V. R. Durante: Università degli studi Roma 3, Dipartimento di Matematica, Largo San Leonardo Murialdo 1, 00146 Roma, Italy; regis@mat.uniroma3.it

P. Vernole: Dipartimento di Matematica, Università degli Studi di Roma "La Sapienza", P. zale Aldo Moro 2, 00185 Roma, Italy.; vernole@mat.uniroma1.it 
by the Cartesian product of a fractal set and a one dimensional interval; suitable Dirichlet energy forms are built taking into account the underlying geometry. From the point of view of PDEs' applications, in all those problems in which the fractal set has both a static and a dynamical role, that is on one side is the boundary of an Euclidean domain and on the other side supports the notion of a Laplacian, as e.g. in transmission problems ([8]), or in Venttsel problems (see e.g. [13]), it is a crucial point to be able to investigate the smoothness properties of the functions in the corresponding energy spaces. In the two-dimensional case one can prove a complete characterization of the energy space on the fractal curve in terms of Lipschitz spaces, which in turn are subsets of Hölder continuous functions on the fractal set (see [4, Theorem 4.6], [14, Theorem 3.1] for the case of Koch curve and [6, Theorem 1] for the case of Sierpiński gasket). In the three-dimensional case, as far as we know, this characterization does not hold. Therefore it is of the utmost importance to approximate the functions in the energy form domains by "smooth" functions. These smoothness properties play a key role in studying the convergence of suitable approximating energy forms to the limit "fractal" one (see e.g. [13] for the two-dimensional case).

In this paper we consider a cylindrical-type fractal mixture surface $S^{(\xi)}=$ $F^{(\xi)} \times I$, which is obtained by the Cartesian product of a snowflake-type mixture $F^{(\xi)}$ and the unit interval $[0,1]$. We define on $S^{(\xi)}$ an energy form $E_{S^{(\xi)}}$ with domain $\mathcal{D}\left(S^{(\xi)}\right)$ (see $\left.(4.2)\right)$.

We denote by $Q^{(\xi)}$ the three-dimensional open bounded cylindrical set whose lateral boundary is $S^{(\xi)}$. On $Q^{(\xi)}$ we introduce the Dirichlet form

$$
E[u]=\int_{Q^{(\xi)}}|D u|^{2} d \mathcal{L}_{3}+E_{S^{(\xi)}}\left[\left.u\right|_{S^{(\xi)}}\right]+\left.\int_{S^{(\xi)}} b|u|_{S^{(\xi)}}\right|^{2} d g,
$$

defined on the space

$$
V\left(Q^{(\xi)}, S^{(\xi)}\right)=\left\{u \in H^{1}\left(Q^{(\xi)}\right),\left.u\right|_{S^{(\xi)}} \in \mathcal{D}\left(S^{(\xi)}\right)\right\}
$$

where $\mathcal{L}_{3}$ is the three-dimensional Lebesgue measure, $E_{S^{(\xi)}}$ is the Dirichlet form defined on $S^{(\xi)}$ (see Section 4 ), $b$ is a continuous and strictly positive function defined on $S^{(\xi)}, g$ is the Hausdorff measure on $S^{(\xi)}$ (see Section 2) and $\left.u\right|_{S^{(\xi)}}$ is the trace to $S^{(\xi)}$ to be properly defined (see Section 3 ).

Our aim is to state density results for the energy spaces $\mathcal{D}\left(S^{(\xi)}\right)$ and $V\left(Q^{(\xi)}, S^{(\xi)}\right)$. In Theorem 5.3 we prove that the space $\mathcal{D}\left(S^{(\xi)}\right)$ has a core, that is a subset dense in $\mathcal{D}\left(S^{(\xi)}\right)$, with respect to the $\mathcal{D}\left(S^{(\xi)}\right)$ norm; this in turn it is a crucial tool together with Proposition 5.5, where we prove an extension result for functions in $\mathcal{D}\left(S^{(\xi)}\right)$, by using the Whitney decomposition. Finally, in Theorem 5.4, we prove that there exists a subset of smooth functions dense in $V\left(Q^{(\xi)}, S^{(\xi)}\right)$.

The plan of the paper is the following: in Section 2 we introduce the Koch mixture $F^{(\xi)}$, the surface $S^{(\xi)}$ and the related measures; in Section 3 we introduce the main relevant functional spaces and we state the trace theorem 
(Theorem 3.5); in Section 4 we introduce the energy form $E[u]$ and its main properties; in Section 5 we state and prove the main density results.

\section{Fractal mixtures}

In this section we recall the definition of scale irregular Koch curves (Koch mixtures), following the construction described in [18].

Let $A=\{1,2\}$ : for $a \in A$, we consider $2<l_{a}<4$, and for each $a \in A$ we set

$$
\Psi^{(a)}=\left\{\psi_{1}^{(a)}, \ldots, \psi_{4}^{(a)}\right\}
$$

the family of contractive similitudes $\psi_{i}^{(a)}: \mathbb{C} \rightarrow \mathbb{C}, i=1, \ldots, 4$, with contraction factor $l_{a}^{-1}$

$$
\begin{aligned}
\psi_{1}^{(a)}(z) & =\frac{z}{l_{a}}, & \psi_{2}^{(a)}(z) & =\frac{z}{l_{a}} e^{i \theta\left(l_{a}\right)}+\frac{1}{l_{a}} \\
\psi_{3}^{(a)}(z) & =\frac{z}{l_{a}} e^{i \theta\left(l_{a}\right)}+\frac{1}{2}+i \sqrt{\frac{1}{l_{a}}-\frac{1}{4}}, & \psi_{4}^{(a)}(z) & =\frac{z-1}{l_{a}}+1
\end{aligned}
$$

where

$$
\theta\left(l_{a}\right)=\arcsin \left(\frac{\sqrt{l_{a}\left(4-l_{a}\right)}}{2}\right)
$$

Let $\Xi=A^{\mathbb{N}}$; we call $\xi \in \Xi$ an environment. We define a left shift $\mathcal{S}$ on $\Xi$ such that if $\xi=\left(\xi_{1}, \xi_{2}, \ldots\right)$, then $\mathcal{S} \xi=\left(\xi_{2}, \xi_{3}, \ldots\right)$. For $\mathcal{O} \subset \mathbb{R}^{2}$, we set

$$
\Phi^{(a)}(\mathcal{O})=\bigcup_{i=1}^{4} \psi_{i}^{(a)}(\mathcal{O}) \text { and } \Phi_{h}^{(\xi)}(O)=\Phi^{\left(\xi_{1}\right)}(O) \circ \ldots \circ \Phi^{\left(\xi_{h}\right)}(O) \text {. }
$$

We consider the line segment of unit length $K$ with endpoints $B=(0,0)$ and $C=(1,0)$. We set, for each $h \in \mathbb{N}, K^{(\xi), h}=\Phi_{h}^{(\xi)}(K): K^{(\xi), h}$ is the $h$-th prefractal curve.

The fractal $K^{(\xi)}$ associated with the environment sequence $\xi$ is defined by

$$
K^{(\xi)}=\overline{\bigcup_{h=1}^{\infty} \Phi_{h}^{(\xi)}(\Gamma)}
$$

where $\Gamma=\{B, C\}$. These fractals don't have any exact self-similarity, but $K^{(\xi)}, \xi \in \Xi$ satisfies

$$
K^{(\xi)}=\Phi^{\left(\xi_{1}\right)}\left(K^{(\delta \xi)}\right) .
$$




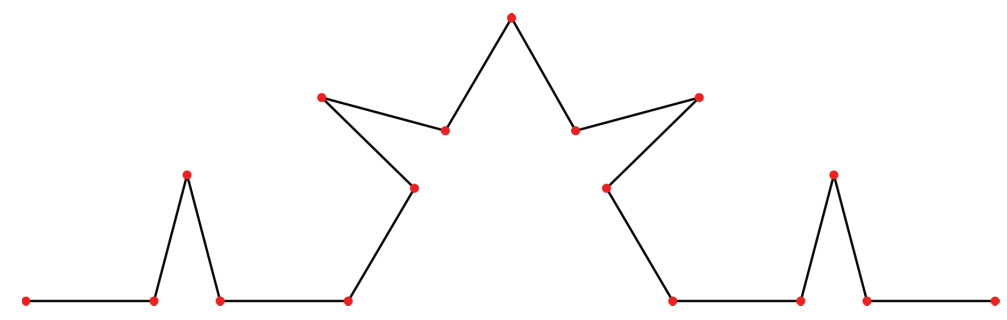

Figure 1: Prefractal mixture curve: $l_{1}=3, l_{2}=2.5$

For $\xi \in \Xi$, we set $i \mid h=\left(i_{1}, i_{2}, \ldots, i_{h}\right)$ and $\psi_{i \mid h}=\psi_{i_{1}}^{\left(\xi_{1}\right)} \circ \ldots \circ \psi_{i_{h}}^{\left(\xi_{h}\right)}$ and for any $\mathcal{O} \subset \mathbb{R}^{2}, \psi_{i \mid h}(\mathcal{O})=\mathcal{O}^{i \mid h}$. There exists a unique Radon measure $\mu^{(\xi)}$ on $K^{(\xi)}$ such that (see $[2$, Section 2])

$$
\mu^{(\xi)}\left(\psi_{i \mid h}\left(K^{\left(\mathcal{S}^{h} \xi\right)}\right)\right)=\frac{1}{4^{h}}
$$

The fractal set $K^{(\xi)}$ and the measure $\mu^{(\xi)}$ depend on the structural constants of the families and the asymptotic frequency of the occurrence of each family. We denote by $c_{a}^{(\xi)}(h)$ the frequency of the occurrence of $a$ in the finite sequence $\left.\xi\right|_{h}, h \geq 1$ :

$$
c_{a}^{(\xi)}(h)=\frac{1}{h} \sum_{i=1}^{h} \mathbf{1}_{\xi_{i}=a}, \quad a=1,2
$$

Let $p_{a}$ be a probability distribution on $A$ and suppose that $\xi$ satisfies

$$
c_{a}^{(\xi)}(h) \rightarrow p_{a}, \quad h \rightarrow \infty,
$$

where $0 \leq p_{a} \leq 1, p_{1}+p_{2}=1$; it also holds

$$
\left|c_{a}^{(\xi)}(h)-p_{a}\right| \leq \frac{f(h)}{h}
$$

$a=1,2(h \geq 1)$, where $f$ is an increasing function on the real line, $f(0)=1$, $f(h) \leq f_{0} h^{\beta_{0}}, f_{0}>1,0 \leq \beta_{0}<1$.

If $\beta_{0}=0$, the measure $\mu^{(\xi)}$ is a $d^{(\xi)}$-measure in the sense of the Definition 3.1, that is there exist two positive constants $C_{1}, C_{2}$, such that

$$
C_{1} r^{d^{(\xi)}} \leq \mu^{(\xi)}\left(B(P, r) \bigcap K^{(\xi)}\right) \leq C_{2} r^{d^{(\xi)}}, \quad \forall P \in K^{(\xi)}
$$

with

$$
d^{(\xi)}=\frac{\ln 4}{p_{1} \ln l_{1}+p_{2} \ln l_{2}}
$$

where $B(P, r)$ denotes the Euclidean ball with center in $P$ and radius $0<r \leq 1$ and $p_{a}$ is the probability distribution on $A$. 
If $\beta_{0}>0$ instead

$$
C_{1} r^{d^{(\xi)}-i} \leq \mu^{(\xi)}\left(B(P, r) \bigcap K^{(\xi)}\right) \leq C_{2} r^{d^{(\xi)}-i}, \quad \forall P \in K^{(\xi)}
$$

We will confine ourselves to the case $\beta_{0}=0$.

Following [4], we introduce the snowflake-type set $F^{(\xi)}$, obtained by the union of three Koch mixtures $K^{(\xi)}$ with the same structural constants, that is

$$
F^{(\xi)}=\bigcup_{i=1}^{3} K_{i}^{(\xi)}
$$

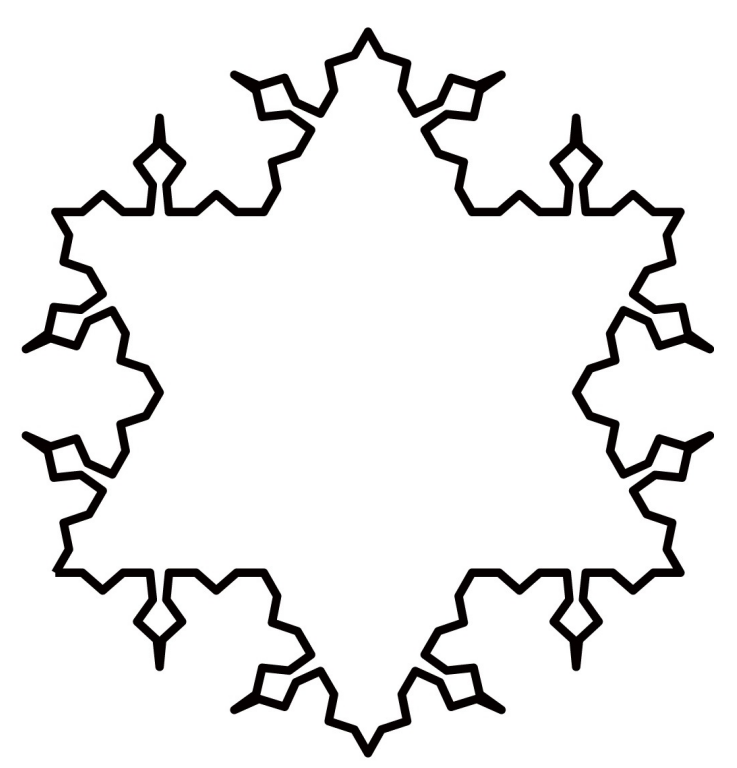

Figure 2: Koch mixture snowflake

and we define a finite Radon measure supported on $F^{(\xi)}$

$$
\mu_{F}^{(\xi)}:=\mu_{1}^{(\xi)}+\mu_{2}^{(\xi)}+\mu_{3}^{(\xi)}
$$

where $\mu_{i}^{(\xi)}$ denotes the normalized $d^{(\xi)}$-dimensional Hausdorff measure restricted to $K_{i}^{(\xi)}, i=1,2,3$.

The dimension of $F^{(\xi)}$ is

$$
D_{f}^{(\xi)}=d^{(\xi)} .
$$

$\Omega^{(\xi)}$ denotes the open bounded two-dimensional domain with boundary $F^{(\xi)}$.

By $S^{(\xi)}$ we denote the cylindrical-type fractal surface

$$
S^{(\xi)}=F^{(\xi)} \times I, \quad \text { where } I=[0,1] .
$$




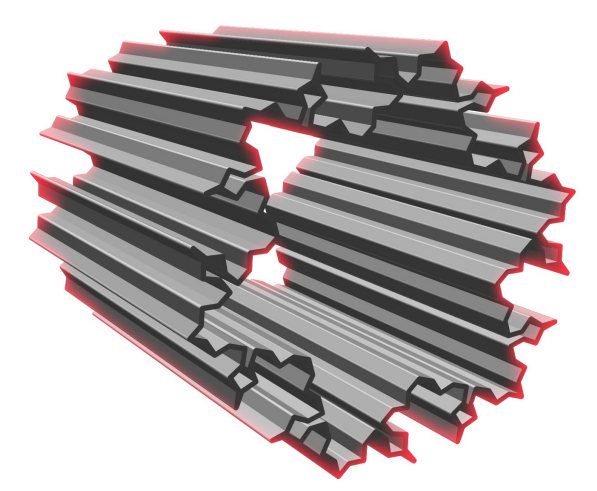

Figure 3: Fractal surface

On $S^{(\xi)}$ we define the following measure

$$
d g^{(\xi)}=d \mu_{F}^{(\xi)} \times d \mathcal{L}_{1}
$$

supported on $S^{(\xi)}$, where $\mathcal{L}_{1}$ is the one dimensional Lebesgue measure on $I$.

By $Q^{(\xi)}$ we denote the open cylindrical domain where $S^{(\xi)}=F^{(\xi)} \times I$ is the "lateral surface" and where the sets $\Omega^{(\xi)} \times\{0\}, \Omega^{(\xi)} \times\{1\}$ are the bases.

We denote by $P \in S^{(\xi)}$, the couple $(x, y)$, where $x=\left(x_{1}, x_{2}\right)$ are the coordinates of the orthogonal projection of $P$ on the plain containing $F^{(\xi)}$ and $y$ is the coordinate of the orthogonal projection of $P$ on the interval $[0,1]$ : $\left(x_{1}, x_{2}\right) \in F^{(\xi)}, y \in I$.

From now on we suppress all the superscripts $\xi$.

\section{Functional spaces}

By $L^{2}(\cdot)$ we denote the Lebesgue space with respect to the Lebesgue measure $\mathcal{L}_{3}$ on subsets of $\mathbb{R}^{3}$, which will be left to the context whenever that does not create ambiguity. Let $T$ be a closed set of $\mathbb{R}^{3}$, by $C(T)$ we denote the space of continuous functions on $T$ and $C^{0, \beta}(T)$ is the space of Hölder continuous functions on $T, 0<\beta<1$. Let $G$ be an open set of $\mathbb{R}^{3}$, by $H^{s}(G), s \in \mathbb{R}^{+}$we denote the Sobolev spaces, possibly fractional (see $[20]$ ). $D(G)$ is the space of infinitely differentiable functions with compact support on $G$.

Definition 3.1. A closed set $M$ is a $d$-set in $\mathbb{R}^{3}(0<d \leq 3)$ if there exist a Borel measure $\mu$ with $\operatorname{supp} \mu=M$ and two positive constants $C_{1}, C_{2}$, such that

$$
C_{1} r^{d} \leq \mu(B(P, r) \bigcap M) \leq C_{2} r^{d}, \quad \forall P \in M
$$

Remark 3.2. F is a $D_{f}$-set. The measure $\mu_{F}$ is a $D_{f}$-measure. $S$ is a $D_{f}+1$-set. The measure $g$ is a $D_{f}+1$-measure. 
Definition 3.3. For $f \in H^{s}(G)$ we put

$$
\gamma_{0} f(P)=\lim _{r \rightarrow 0} \frac{1}{|B(P, r) \bigcap G|} \int_{B(P, r) \cap G} f(\mathcal{P}) d \mathcal{L}_{3}
$$

at every point $P \in \bar{G}$ where the limit exists.

We now define the Besov space on $S$ : we recall here the definition which best fits our aims and we restrict ourselves to the case $p=q=2$ and $\beta=\frac{D_{f}}{2}$; for a general treatment see [7].

Definition 3.4. We say that $f \in B_{\frac{D_{f}}{2}}^{2,2}(S)$ if $f \in L^{2}(S, g)$ and it holds

$$
\|f\|_{\frac{B_{\frac{D_{f}}{2}}^{2,2}(S)}{2}<+\infty}<
$$

where

$$
\|f\|_{B_{\frac{D_{f}}{2}}^{2,2}(S)}=\|f\|_{L^{2}(S, g)}+\left(\iint_{\left|P-P^{\prime}\right|<1} \frac{\left|f(P)-f\left(P^{\prime}\right)\right|^{2}}{\left|P-P^{\prime}\right|^{2 D_{f}+1}} d g(P) d g\left(P^{\prime}\right)\right)^{\frac{1}{2}}
$$

Theorem 3.5. $B_{\frac{D_{f}}{2}}^{2,2}(S)$ is the trace space of $H^{1}(Q)$ that is:

(1) There exists a linear and continuous operator $\gamma_{0}: H^{1}(Q) \rightarrow B_{\frac{D_{f}}{2}}^{2,2}(S)$.

(2) There exists a linear and continuous operator Ext $: B_{\frac{D_{f}}{2}}^{2,2}(S) \rightarrow H^{1}(Q)$, such that $\gamma_{0} \circ$ Ext is the identity operator on $B_{\frac{D_{f}}{2}}^{2,2}(S)$, that is

$$
\gamma_{0} \circ \operatorname{Ext}=I d_{\frac{B_{\frac{D_{f}}{2}}^{2,2}(S)}{}} .
$$

In the following we denote by the symbol $\left.u\right|_{S}$ the trace $\gamma_{0} u$ to $S$.

\section{Energy forms}

By proceeding as in [4] we construct an energy form on $F$, by defining a Lagrangian measure $\mathcal{L}_{F}$ on $F$, which has the role of Euclidean Lagrangian $d \mathcal{L}(u, v)=\nabla u \nabla v d x$. The corresponding energy form on $F$ is given by

$$
\mathcal{E}_{F}(u, v)=\int_{F} d \mathcal{L}_{F}(u, v)
$$

with domain $\mathcal{D}(F)=\left\{u \in L^{2}\left(F, \mu_{F}\right): \mathcal{E}_{F}[u]<+\infty\right\}$ dense in $L^{2}\left(F, \mu_{F}\right)$. 
Proposition 4.1. $\mathcal{D}(F)$ is a Hilbert space equipped with the following norm

$$
\|u\|_{\mathcal{D}(F)}=\left(\|u\|_{L^{2}(F)}^{2}+\mathcal{E}_{F}[u]\right)^{\frac{1}{2}} .
$$

As in [16] it can be proved that

Proposition 4.2. $\mathcal{D}(F)$ is embedded in $C^{0, \beta}(F)$, with $\beta=\frac{\ln 4}{2 \ln \left(\min \left(l_{1}, l_{2}\right)\right)}$.

We now define the energy form on $S$ and the fractal Laplacian $\Delta_{S}$.

$$
E_{S}[u]=\int_{I} \mathcal{E}_{F}[u] \mathrm{d} \mathcal{L}_{1}+\int_{F} \int_{I}\left|D_{y} u\right|^{2} \mathrm{~d} \mathcal{L}_{1} d \mu_{F},
$$

The form $E_{S}$ is defined for $u \in \mathcal{D}(S)$,

$$
\mathcal{D}(S)=\overline{C(S) \cap L^{2}([0,1] ; \mathcal{D}(F)) \cap H^{1}\left([0,1] ; L^{2}(F)\right)} \|^{\|\cdot\|_{\mathcal{D}(S)}},
$$

where $\|\cdot\|_{\mathcal{D}(S)}$ is the intrinsic norm

$$
\|u\|_{\mathcal{D}(S)}=\left(E_{S}[u]+\|u\|_{L^{2}(S, g)}^{2}\right)^{\frac{1}{2}} .
$$

Proposition 4.3. $E_{S}(u, v)$ with domain $\mathcal{D}(S) \times \mathcal{D}(S)$ is a closed bilinear form in $L^{2}(S, g)$ and $\mathcal{D}(S)$ is a Hilbert space equipped with the intrinsic norm.

From Proposition 4.3 we have

Theorem 4.4. There exists a unique non positive self-adjoint operator $\Delta_{S}$ on $L^{2}(S, g)$ with domain $\mathcal{D}\left(\Delta_{S}\right):=\left\{u \in L^{2}(S, g): \Delta_{S} u \in L^{2}(S, g)\right\} \subseteq \mathcal{D}(S)$ dense in $L^{2}(S, g)$ such that

$$
E_{S}(u, v)=-\int_{S} \Delta_{S} u v d g, \quad \text { for each } u \in \mathcal{D}\left(\Delta_{s}\right), v \in \mathcal{D}(S) .
$$

We now give an embedding result for the domain $\mathcal{D}(S)$. Unlike the two dimensional case where there is a characterization of the functions in $\mathcal{D}(F)$ in terms of the so-called Lipschitz spaces (see [14, Theorem 3.1]), for $\mathcal{D}(S)$ we do not have a characterization, but the following result holds:

Proposition 4.5. $\mathcal{D}(S)$ is embedded in $B_{\beta}^{2,2}(S)$, for any $0<\beta<1$.

Proof. We follow the proof in [8], adapted to the present case. We recall that

$$
\mathcal{D}(S):={\overline{C(S) \bigcap L^{2}([0,1] ; \mathcal{D}(F)) \bigcap H^{1}\left([0,1] ; L^{2}(F)\right)}}^{\|\cdot\|_{\mathcal{D}(S)}}
$$

Following [17] we define $B_{D_{f}-\varepsilon, 1}^{2,2}(S):=L^{2}\left([0,1] ; B_{D_{f}-\varepsilon}^{2,2}(F)\right) \bigcap H^{1}\left([0,1] ; L^{2}(F)\right)$ for $\varepsilon>0$. 
For any Banach space $X$ and for any $0<\beta<1, H^{1}([0,1] ; X) \subset H^{\beta}([0,1] ; X)$, moreover if $p=q=2$ and $\beta$ is not integer, it holds

$$
H^{\beta}([0,1] ; X) \equiv B_{\beta}^{2,2}([0,1] ; X)
$$

Hence if $0<\beta<1$

$$
\begin{aligned}
B_{D_{f}-\varepsilon, 1}^{2,2}(S) & \subset L^{2}\left([0,1] ; B_{D_{f}-\varepsilon}^{2,2}(F)\right) \bigcap B_{\beta}^{2,2}\left(0,1 ; L^{2}(F)\right) \\
& \subset L^{2}\left([0,1] ; B_{\beta}^{2,2}(F)\right) \bigcap B_{\beta}^{2,2}\left([0,1] ; L^{2}(F)\right)=B_{\beta}^{2,2}(S),
\end{aligned}
$$

the last equivalence can be proved following [17].

Now we introduce the energy form on $Q$. Let us consider the space

$$
V(Q, S)=\left\{u \in H^{1}(Q):\left.u\right|_{S} \in \mathcal{D}(S)\right\}
$$

Let $b$ be a continuous and strictly positive function on $S$. We consider the energy form $E$

$$
E[u]=\int_{Q}|D u|^{2} d \mathcal{L}_{3}+E_{S}\left[\left.u\right|_{S}\right]+\left.\int_{S} b|u|_{S}\right|^{2} d g
$$

defined on $V(Q, S)$. From now on we denote by $L^{2}(\bar{Q}, m)$ the Lebesgue space with respect to the measure

$$
d m=d \mathcal{L}_{3}+d g
$$

By $E(u, v)$ we denote the bilinear form

$$
E(u, v)=\int_{Q} D u D v d \mathcal{L}_{3}+E_{S}\left(\left.u\right|_{S},\left.v\right|_{S}\right)+\left.\left.\int_{S} b u\right|_{S} v\right|_{S} d g
$$

defined on $V(Q, S) \times V(Q, S)$.

Proposition 4.6. The form $E$ is a Dirichlet form on $L^{2}(\bar{Q}, m)$ and $V(Q, S)$ is a Hilbert space equipped with the scalar product

$$
(u, v)_{V(Q, S)}=(u, v)_{H^{1}(Q)}+E_{S}(u, v)+(u, v)_{L^{2}(S, g)}
$$

with norm

$$
\|u\|_{V(Q, S)}=\left(\|u\|_{H^{1}(Q)}^{2}+\|u\|_{\mathcal{D}(S)}^{2}\right)^{\frac{1}{2}} .
$$




\section{Density theorems}

5.1. Density theorem for $\mathcal{D}(S)$. In the notations of $[17$, p. 8$]$, by $W(0,1)$ we denote the following space:

$$
W(0,1):=L^{2}([0,1] ; \mathcal{D}(F)) \bigcap H^{1}\left([0,1] ; L^{2}(F)\right) .
$$

This is a Hilbert space equipped with the norm

$$
\|u\|_{W(0,1)}=\left(\|u\|_{L^{2}([0,1] ; \mathcal{D}(F))}^{2}+\left\|D_{y} u\right\|_{L^{2}\left([0,1] ; L^{2}(F)\right)}^{2}\right)^{\frac{1}{2}} .
$$

From [17, p. 11, Theorem 2.1], the following result holds

Proposition 5.1. The space $D([0,1] ; \mathcal{D}(F))$ is densely embedded in $W(0,1)$, that is

$$
\overline{D([0,1] ; \mathcal{D}(F))^{\|\cdot\|_{W(0,1)}}=W(0,1) .}
$$

We now prove that

Proposition 5.2. $D([0,1] ; \mathcal{D}(F))$ is embedded in $C(S)$.

Proof. From Proposition 4.2 it holds that $\mathcal{D}(F) \subset C^{0, \beta}(F)$, in particular $\mathcal{D}(F) \subset$ $C(F)$, then

$$
D([0,1] ; \mathcal{D}(F)) \subset C([0,1] ; \mathcal{D}(F)) \subset C([0,1] ; C(F)) .
$$

It remains to be proved

$$
C([0,1] ; C(F)) \equiv C(S)
$$

Following [3, pp. 68-70], if $u \in C(S)$, this implies that $u(\cdot, y) \in C(F)$ for every $y \in[0,1], u(x, \cdot) \in C([0,1])$ for every $x \in F$, and that $\sup _{y \in[0,1]} \sup _{x \in F}|u(x, y)|<\infty$, then

$$
C(S) \subseteq C([0,1] ; C(F))
$$

If $u \in C([0,1] ; C(F))$, then $u(\cdot, y) \in C(F)$ for every fixed $y \in[0,1]$, and from the continuity of $u$ in $[0,1]$, for every $x \in F$, it follows that

$$
\sup _{x \in F}\left|u(x, y)-u\left(x, y_{n}\right)\right| \rightarrow 0
$$

for every $\left\{y_{n}\right\} \subset I, y_{n} \rightarrow y$ when $n \rightarrow \infty$. Therefore $C([0,1] ; C(F)) \equiv C(S)$.

Theorem 5.3. The space $D([0,1] ; \mathcal{D}(F))$ is dense in $\mathcal{D}(S)$ with respect to the intrinsic norm $\|\cdot\|_{\mathcal{D}(S)}$. 
Proof. From Proposition 5.2 and (4.1) it holds that

$$
D([0,1] ; \mathcal{D}(F)) \subset C(S) \bigcap L^{2}([0,1] ; \mathcal{D}(F)) \bigcap H^{1}\left([0,1] ; L^{2}(F)\right)
$$

which amounts to say that $D([0,1] ; \mathcal{D}(F)) \subset C(S) \bigcap W(0,1)$; from the definition of $\mathcal{D}(S)$ we have

$$
C(S) \bigcap W(0,1) \subset \mathcal{D}(S)
$$

It follows that

$$
D([0,1] ; \mathcal{D}(F)) \subset \mathcal{D}(S) .
$$

Now let $f$ be a function in $\mathcal{D}(S)$, then from the definition of $\mathcal{D}(S)$ it follows that there exists $\left\{\varphi_{n}\right\} \subset W(0,1) \bigcap C(S)$ such that

$$
\left\|\varphi_{n}-f\right\|_{\mathcal{D}(S)} \rightarrow 0 \text { for } n \rightarrow \infty \text {. }
$$

On the other hand $\varphi_{n} \in W(0,1)$, and from [17, p. 11, Theorem 2.1] , there exists $\left\{\psi_{m, n}\right\}_{m \in \mathbb{N}} \subset D([0,1] ; \mathcal{D}(F))$ such that, for every fixed $n$

$$
\left\|\psi_{m, n}-\varphi_{n}\right\|_{W(0,1)} \rightarrow 0 \quad \text { when } m \rightarrow \infty
$$

From Fubini Theorem for measure valued functions it follows that $\|\cdot\|_{\mathcal{D}(S)}=$ $\|\cdot\|_{W(0,1)}$ and hence for every fixed $n$

$$
\left\|\psi_{m, n}-\varphi_{n}\right\|_{\mathcal{D}(S)} \rightarrow 0 \quad \text { for } m \rightarrow \infty
$$

We now use a diagonalization argument. From [1, Corollary 1.16] there exists an increasing mapping

$$
m \rightarrow n(m)
$$

that tends to $\infty$ for $m \rightarrow \infty$, such that

$$
\limsup _{m \rightarrow \infty}\left\|\psi_{m, n(m)}-\varphi_{n(m)}\right\|_{\mathcal{D}(S)} \leq \limsup _{n \rightarrow \infty} \lim _{m \rightarrow \infty}\left\|\psi_{m, n}-\varphi_{n}\right\|_{\mathcal{D}(S)}
$$

The right hand side of (5.1) tends to zero when $\mathrm{m} \rightarrow \infty$ and from this it follows that $\lim \sup _{m \rightarrow \infty}\left\|\psi_{m, n(m)}-\varphi_{n(m)}\right\|_{\mathcal{D}(S)}=0$. Hence also

$$
\liminf _{m \rightarrow \infty}\left\|\psi_{m, n(m)}-\varphi_{n}(m)\right\|_{\mathcal{D}(S)}=0 .
$$

This proves that $\lim _{m \rightarrow \infty}\left\|\psi_{m, n(m)}-\varphi_{n(m)}\right\|_{\mathcal{D}(S)}=0$.

Finally $\left\|\psi_{n(m), m}-f\right\|_{\mathcal{D}(S)} \leq\left\|\psi_{n(m), m}-\varphi_{n(m)}\right\|_{\mathcal{D}(S)}+\left\|\varphi_{n(m)}-f\right\|_{\mathcal{D}(S)} \rightarrow 0$ for $m \rightarrow \infty$. 
5.2. Density theorem for $V(Q, S)$. We now state the main theorem of the section. Let $Q, S$ and $V(Q, S)$ be defined as in Section 2 and Section 4 respectively.

Theorem 5.4. For every $u \in V(Q, S)$, there exists $\left\{\psi_{n}\right\} \subset V(Q, S) \bigcap C(\bar{Q})$ such that:

(1) $\left\|\psi_{n}-u\right\|_{H^{1}(Q)} \rightarrow 0$, for $n \rightarrow \infty$

(2) $\left\|\psi_{n}-u\right\|_{L^{2}(\bar{Q}, m)} \rightarrow 0$, for $n \rightarrow \infty$

(3) $E_{S}\left[\psi_{n}-u\right] \rightarrow 0$, for $n \rightarrow \infty$.

In order to prove this theorem, we need a preliminary proposition on trace and extension operators.

Proposition 5.5. Let $\beta=\frac{D_{f}}{2}$. Let $\gamma_{0}$ and Ext be the trace and the extension operator defined in Theorem 3.5, respectively. Then

(1) If $u \in C\left(\mathbb{R}^{3}\right) \bigcap H^{1}\left(\mathbb{R}^{3}\right)$ then $\gamma_{0} u \in C(S) \bigcap B_{\beta}^{2,2}(S)$.

(2) If $u \in C(S) \bigcap B_{\beta}^{2,2}(S)$ then $\operatorname{Ext}(u) \in C\left(\mathbb{R}^{3}\right) \bigcap H^{1}\left(\mathbb{R}^{3}\right)$.

Proof. We start proving (1). Since $u \in H^{1}\left(\mathbb{R}^{3}\right)$, then for $P \in S, \gamma_{0} u(P)$ exists and, from Theorem 3.5, $\gamma_{0} u$ belongs to $B_{\beta}^{2,2}(S)$ with $\beta=\frac{D_{f}}{2}$; since $u$ is also in $C\left(\mathbb{R}^{3}\right)$, in particular $u$ is in $C(S)$. By the Mean Value Theorem there exists $\xi \in B(P, r) \bigcap S$ such that

$$
\frac{1}{m(B(P, r) \bigcap S)} \int_{B(P, r) \cap S} u(\mathcal{P}) d \mathcal{L}_{3}=u(\xi) .
$$

Hence when $r \rightarrow 0$

$$
u(\xi) \rightarrow u(P) .
$$

In order to prove (2) we make use of Whitney decomposition. We refer to [7, p. 23] for details. Let $Q_{i}$ be the cubes in $\mathbb{R}^{3} \backslash S$ such that $\bigcup_{i} Q_{i}=\mathbb{R}^{3} \backslash S$, with centers $P_{i}, l_{i}=\operatorname{diam} Q_{i}$ and $\left\{\phi_{i}\right\}$ the associated unity partition. From [7, p. 109], we define for $P \in \mathbb{R}^{3} \backslash S$

$$
\operatorname{Ext}(u)(P)=\sum_{i \in I} \phi_{i}(P) c_{i} \int_{\left|t-P_{i}\right| \leq 6 l_{i}} u(t) d g(t),
$$

where $c_{i}=\left(g\left(\left|t-P_{i}\right| \leq 6 l_{i}\right)\right)^{-1}$.

In our assumptions $u \in B_{\beta}^{2,2}(S)$, then from Theorem 3.5, $\operatorname{Ext}(u) \in H^{1}\left(\mathbb{R}^{3}\right)$ and $\gamma_{0}(\operatorname{Ext}(u))=u$ on $S$. It results, by construction, that $\operatorname{Ext}(u)$ is in particular continuous in $\mathbb{R}^{3} \backslash S$. Since $u \in C(S) \bigcap B_{\beta}^{2,2}(S)$, it remains to prove that for every $P_{0} \in S$

$$
\left|\operatorname{Ext}(u)(P)-u\left(P_{0}\right)\right| \rightarrow 0 \quad \text { when } P \rightarrow P_{0},
$$

that is for every $\varepsilon>0 \exists \delta_{\varepsilon}:\left|P-P_{0}\right|<\delta_{\varepsilon}$, then $\left|\operatorname{Ext}(u)(P)-u\left(P_{0}\right)\right|<\varepsilon$. 
We now estimate $\left|\operatorname{Ext}(u)(P)-u\left(P_{0}\right)\right|$.

$$
\begin{aligned}
\left|\operatorname{Ext}(u)(P)-u\left(P_{0}\right)\right| & =\left|\sum_{i \in I} \phi_{i}(P) c_{i} \int_{\left|t-P_{i}\right| \leq 6 l_{i}} u(t) d g-u\left(P_{0}\right)\right| \\
& =\left|\sum_{i \in I} \phi_{i}(P) c_{i} \int_{\left|t-P_{i}\right| \leq 6 l_{i}}\left(u(t)-u\left(P_{0}\right)\right) d g\right| \\
& \leq c\left(l_{i} \frac{-\frac{-\left(D_{f}+1\right)}{2}}{2}\left(\int_{\left|t-P_{i}\right| \leq 6 l_{i}}\left|u(t)-u\left(P_{0}\right)\right|^{2} d g\right)^{\frac{1}{2}}\right.
\end{aligned}
$$

where the last inequality is obtained from Hölder inequality and from now $c$ will denote possibly different constants. Since $g$ is a $\left(D_{f}+1\right)$-measure supported on $S$ and since $\left|P-P_{0}\right| \leq \delta$, we obtain

$$
\begin{aligned}
& c\left(l_{i}\right)^{\frac{-\left(D_{f}+1\right)}{2}}\left(\int_{\left|t-P_{i}\right| \leq 6 l_{i}}\left|u(t)-u\left(P_{0}\right)\right|^{2} d g\right)^{\frac{1}{2}} \\
& =c\left(l_{i}\right)^{\frac{-\left(D_{f}+1\right)}{2}}\left(\int_{\left\{\left|t-P_{i}\right| \leq 6 l_{i}\right\} \cap\left\{\left|t-P_{0}\right| \leq \delta\right\}}\left|u(t)-u\left(P_{0}\right)\right|^{2} d g\right)^{\frac{1}{2}} .
\end{aligned}
$$

As $u \in C(S)$ we get

$$
\begin{aligned}
& c\left(l_{i}\right)^{\frac{-\left(D_{f}+1\right)}{2}}\left(\int_{\left\{\left|t-P_{i}\right| \leq 6 l_{i}\right\} \cap\left\{\left|t-P_{0}\right| \leq \delta\right\}}\left|u(t)-u\left(P_{0}\right)\right|^{2} d g\right)^{\frac{1}{2}} \\
& \leq c\left(l_{i}\right)^{\frac{-\left(D_{f}+1\right)}{2}} \sup _{\left\{\left|(x, y)-P_{i}\right| \leq 6 l_{i}\right\} \bigcap\left\{\left|(x, y)-P_{0}\right| \leq \delta\right\}}\left|u(x, y)-u\left(P_{0}\right)\right|\left(\int_{\left\{\left|t-P_{i}\right| \leq 6 l_{i}\right\} \cap\left\{\left|t-P_{0}\right| \leq \delta\right\}} d g\right)^{\frac{1}{2}} \\
& \leq c l_{i}^{\frac{-\left(D_{f}+1\right)}{2}} l_{i}^{\frac{D_{f}+1}{2}} \varepsilon=c \varepsilon
\end{aligned}
$$

where the last inequality follows from the continuity of $u$ on $S$.

We are now ready to prove Theorem 5.4.

Proof of Theorem 5.4. We start proving (1). Let us consider $u \in V(Q, S)$, then $\left.u\right|_{S} \in \mathcal{D}(S)$. From Theorem 5.3 there exists $\left\{\varphi_{n}\right\} \subset D([0,1] ; \mathcal{D}(F))$ such that

$$
\left\|\varphi_{n}-\left.u\right|_{S}\right\|_{\mathcal{D}(S)} \rightarrow 0, \quad \text { when } n \rightarrow \infty
$$

Let $\widehat{\varphi_{n}}$ be the function defined as $\operatorname{Ext}\left(\varphi_{n}\right)$ and let $\widehat{u}$ be the function defined as $\operatorname{Ext}\left(\left.u\right|_{S}\right)$. Then from Proposition $5.5 \widehat{\varphi_{n}} \in H^{1}(Q) \bigcap C(\bar{Q})$ and $\widehat{u} \in H^{1}(Q)$ (see [7]). 
We prove that $\left\|\widehat{\varphi_{n}}-\widehat{u}\right\|_{H^{1}(Q)} \rightarrow 0$; in fact from Theorem 3.5 and the inclusion of $\mathcal{D}(S)$ in $B_{\frac{D_{f}}{2}}^{2,2}(S)$ (see Proposition 4.5),

$$
\left\|\widehat{\varphi_{n}}-\widehat{u}\right\|_{H^{1}(Q)} \leq c\left\|\varphi_{n}-\left.u\right|_{S}\right\|_{B_{\frac{D_{f}}{2}}^{2,2}(S)} \leq c\left\|\varphi_{n}-\left.u\right|_{S}\right\|_{\mathcal{D}(S)}
$$

From the density Theorem 5.3, $\left\|\widehat{\varphi_{n}}-\widehat{u}\right\|_{H^{1}(Q)} \rightarrow 0$.

Now let us consider $u-\widehat{u}$ : this is a function in $H^{1}(Q)$ and $\left.(u-\widehat{u})\right|_{S}=0$, then $u-\widehat{u} \in H_{0}^{1}(Q)$, (see $\left[23\right.$, Theorem 3]); there exists $\left\{\eta_{m}\right\}_{m \in \mathbb{N}} \subset C_{0}^{1}(\bar{Q})$ such that

$$
\left\|\eta_{m}-(u-\widehat{u})\right\|_{H^{1}(Q)} \rightarrow 0 .
$$

Let $\left\{\psi_{n, m}\right\}$ denote the doubly indexed sequence of function $\left\{\widehat{\varphi_{n}}-\eta_{m}\right\}$. The sequence $\left\{\psi_{n, m}\right\} \subset H^{1}(Q) \bigcap C(\bar{Q})$. From [1, Corollary 1.16] we deduce that $\left\{\psi_{m, n}\right\}$ converges to $u$ in $H^{1}(Q)$ as $n \rightarrow \infty$. In fact there exists an increasing mapping $n \rightarrow m(n)$, tending to $\infty$ as $n \rightarrow \infty$, such that

$$
\begin{aligned}
\limsup _{n \rightarrow \infty}\left\|u-\psi_{n, m(n)}\right\|_{H^{1}(Q)} & =\limsup _{n \rightarrow \infty}\left\|u-\widehat{\varphi_{n}}-\eta_{m(n)}\right\|_{H^{1}(Q)} \\
& \leq \limsup _{n \rightarrow \infty}\left(\left\|u-\widehat{u}-\eta_{m(n)}\right\|_{H^{1}(Q)}+\left\|\widehat{\varphi_{n}}-\widehat{u}\right\|_{H^{1}(Q)}\right),
\end{aligned}
$$

then by applying $[1$, Corollary 1.16] to the right hand side of the above inequality it follows that

$$
\limsup _{n \rightarrow \infty}\left\|u-\psi_{n, m(n)}\right\|_{H^{1}(Q)} \leq \lim _{m \rightarrow \infty} \lim _{n \rightarrow \infty}\left\{\left\|u-\widehat{u}-\eta_{m}\right\|_{H^{1}(Q)}+\left\|\widehat{\varphi_{n}}-\widehat{u}\right\|_{H^{1}(Q)}\right\} .
$$

The two terms in the sum tend to 0 when $m, n \rightarrow \infty$, then

$$
\limsup _{n \rightarrow \infty}\left\|\psi_{n, m(n)}-u\right\|_{H^{1}(Q)}=0,
$$

and also $\liminf \operatorname{in}_{n \rightarrow \infty}\left\|\psi_{n, m(n)}-u\right\|_{H^{1}(Q)}=0$, hence we conclude that

$$
\left\|\psi_{n, m(n)}-u\right\|_{H^{1}(Q)} \rightarrow 0, \quad n \rightarrow \infty .
$$

From now on we use the abbreviation

$$
\psi_{n}=\psi_{n, m(n)} .
$$

Now we prove (2), that is

$$
\left\|\psi_{n}-u\right\|_{L^{2}(Q, m)}=\left\|\psi_{n}-u\right\|_{L^{2}(Q)}+\left\|\psi_{n}-u\right\|_{L^{2}(S)} \rightarrow 0 .
$$

The first term in (5.2) tends to 0 when $n \rightarrow \infty$ since

$$
\left\|\psi_{n}-u\right\|_{L^{2}(Q)} \leq\left\|\psi_{n}-u\right\|_{H^{1}(Q)} .
$$


We now prove that the second term in (5.10) tends to 0.

$$
\left\|\psi_{n}-u\right\|_{L^{2}(S)}=\left\|\left.\widehat{\varphi_{n}}\right|_{S}-\left.\eta_{n}\right|_{S}-\left.u\right|_{S}\right\|_{L^{2}(S)} \equiv\left\|\varphi_{n}-\left.u\right|_{S}\right\|_{L^{2}(S)} \leq\left\|\varphi_{n}-\left.u\right|_{S}\right\|_{\mathcal{D}(S)},
$$

and the last quantity tends to zero from the density of $D([0,1] ; \mathcal{D}(F))$ in $\mathcal{D}(S)$. This proves that $\psi_{n} \rightarrow u$ in $L^{2}(\bar{Q}, m)$.

Now we prove (3):

$$
E_{S}\left[\left.\left(u-\psi_{n}\right)\right|_{S}\right]=E_{S}\left[\left.u\right|_{S}-\left.\psi_{n}\right|_{S}\right] \equiv E_{S}\left[\left.u\right|_{S}-\varphi_{n}\right] \leq\left\|\left.u\right|_{S}-\varphi_{n}\right\|_{\mathcal{D}(S)} \rightarrow 0 .
$$

\section{References}

[1] Attouch, H., Variational Convergence for Functions and Operators. Appl. Math. Ser. Boston (MA): Pitman 1984.

[2] Barlow, M. T. and Hambly, B. M., Transition density estimates for Brownian motion on scale irregular Sierpinski gaskets. Ann. Inst. Henri Poincaré Probab. Statist. 33 (1997)(5), $531-557$.

[3] Brezzi, F. and Gilardi, G., Fundamentals of PDE for numerical analysis. In: Finite Element Handbook (eds.: H. Kardenstuncer et al.). New York: McGrawHill 1987.

[4] Freiberg, U. and Lancia, M. R., Energy form on a closed fractal curve. Z. Anal. Anwend. 23 (2004)(1), 115 - 135.

[5] Fukushima, M., Dirichlet forms, diffusion processes and spectral dimension for nested fractals. In: Ideas and Methods in Mathematical Analysis, Stochastics, and Applications (Proceedings Oslo 1988; eds:. S. Albeverio et al.). Cambridge: Cambridge Univ. Press 1992, pp. $151-161$.

[6] Jonsson, A., Brownian motion on fractals and function spaces. Math. Z. 6 (1996), $495-504$.

[7] Jonsson, A. and Wallin, H., Function Spaces on Subset of $\mathbb{R}^{n}$. Math. Rep. 2. London: Harwood Acad. Publ. 1984.

[8] Lancia, M. R., Second order transmission problems across a fractal surface. Rend. Accad. Naz. Sci. XL Mem. Mat. Appl. (5) 27 (2003), $191-213$.

[9] Lancia, M. R. and Vernole, P., Convergence results for parabolic transmission problems across higly conductive layers with small capacity. Adv. Math. Sci. Appl. 16 (2006)(2), $411-445$.

[10] Lancia, M. R. and Vernole, P., Irregular heat flow problems. SIAM J. Math. Anal. 42 (2010), 1539 - 1567.

[11] Lancia, M. R. and Vernole, P., Semilinear evolution transmission problems across fractal layers. Nonlinear Anal. 75 (2012), 4222 - 4240.

[12] Lancia, M. R. and Vernole, P., Semilinear fractal problems: approximation and regularity results. Nonlinear Anal. 80 (2013), 216 - 232. 
[13] Lancia, M. R. and Vernole, P., Venttsel' problems in fractal domains. J. Evol. Equ. 3 (2014), 681 - 712.

[14] Lancia, M. R. and Vivaldi, M. A., Lipschitz spaces and Besov traces on self similar fractals. Rend. Accad. Naz. Sci. XL Mem. Mat. Appl. (5) 23 (1999), $101-116$.

[15] Lancia, M. R. and Vivaldi, M. A., Asymptotic convergence of transmission energy forms. Adv. Math. Sc. Appl. 13 (2003), 315 - 341.

[16] Liang, H., On the construction of certain fractal mixtures. Thesis in Appl. Math., Worcester Polytechnic Institute 2009.

[17] Lions, J. and Magenes, E., Non-Homogeneous Boundary Valued Problems and Applications. Vol. I. Berlin: Springer 1972.

[18] Mosco, U., Harnack inequalities on scale irregular Sierpinski gaskets. In: Nonlinear Problems in Mathematical Physics and Related Topics. II (in honor of Professor O. A. Ladyzhenskaya; eds.: M. Sh. Birman et al.). New York: Kluwer Acad. 2002, pp. $305-328$.

[19] Mosco, U. and Vivaldi, M. A., Variational problems with fractal layers. Rend. Acc. Naz. Sci. XL Mem. Mat. Appl. (5) 27 (2003), $247-251$.

[20] Nečas, J., Les Méthodes Directes en Théorie des Équations Elliptiques (in French). Paris: Masson 1967.

[21] Sapoval, B., General formulation of Laplacian transfer across irregular surfaces. Phys. Rev. Lett. 73 (1994), $3314-3316$.

[22] Shinbrot, M., Water waves over periodic bottoms in three dimensions. J. Inst. Math. Appl. 25 (1980)(4), $367-385$.

[23] Wallin, H., The trace to the boundary of Sobolev spaces on a snowflake. Manuscripta Math. 73 (1991), $117-125$.

Received July 17, 2014; revised March 9, 2015 